\title{
Fatal Acute Liver Failure With Intravenous Amiodarone: A Case Report and Literature Review
}

\author{
Vijay Gayamª, c, Mazin Khalid ${ }^{\mathrm{a}}$, Sumit Dahal ${ }^{\mathrm{a}}$, Pavani Garlapatia ${ }^{\mathrm{a}}$ Arshpal Gilla, b, \\ Ragin Alex ${ }^{\mathrm{a}, \mathrm{b}}$, Mohammad Mansour ${ }^{\mathrm{a}}$
}

\begin{abstract}
Amiodarone is a drug which frequently causes elevated transaminases. However, acute liver failure has been rarely reported. Here, we present a case of fatal acute liver failure following the administration of intravenous amiodarone. It is important to be aware of this rare but potentially fatal complication of intravenous amiodarone so that it can be withdrawn immediately at the first sign of hepatic impairment.
\end{abstract}

Keywords: Amiodarone; Liver; Failure

\section{Introduction}

Amiodarone is commonly used in the management of arrhythmias. Its lipophilic structure enables it to remain in the body for up to 6 months. It has a well-documented toxicity profile with rash, fatigue, tremor and pulmonary fibrosis being the most common side effects. Liver toxicity is also commonly seen and elevation of transaminases with long-term amiodarone use is reported in $15 \%$ to $50 \%$ of the cases [1]. However, cases of acute liver failure have rarely been reported. Here we present a case of fatal acute liver failure induced by intravenous amiodarone.

\section{Case Report}

A 53-year-old African American male was referred to the emergency department from his dialysis center following an episode of tachycardia. Past medical history was notable for end stage renal disease, hypertension, coronary artery disease and atrial fibrillation. The patient stated feeling fatigued for 1 month but denied any other complaint. He denied fever, headache, dizziness, shortness of breath, chest pain, diaphoresis,

Manuscript submitted October 11, 2017, accepted January 24, 2018

anterfaith Medical Center, 1545 Atlantic Ave, Brooklyn, NY 11213, USA

${ }^{\mathrm{b}}$ American University of Antigua, Jabberwock Rd, Osbourn, Antigua and Barbuda

${ }^{c}$ Corresponding Author: Vijay Gayam, Interfaith Medical Center, 1545 Atlantic Ave, Brooklyn, NY 11213, USA. Email: vgayam@interfaithmedical.com abdominal pain, nausea, vomiting, diarrhea, constipation, difficulty swallowing, temperature intolerance nor skin changes. On admission, his pulse was irregular at 150 beats per minute and his blood pressure was $136 / 95 \mathrm{~mm} \mathrm{Hg}$. The patient was not in any apparent distress and was oriented to person, place and time. His physical examination was remarkable for decreased breath sounds and rales in the right lower lobe of the lung, and bilateral lower extremity edema. There were no signs of icterus, palmar erythema, spider angioma, abdominal ascites, tenderness nor organomegaly. Electrocardiogram revealed atrial flutter and he was started on intravenous diltiazem drip.

On the second day of hospitalization, the patient started having cough and sharp chest pain without fever or shortness of breath. He continued to have atrial flutter, which was not rate-controlled, and was thus started on intravenous infusion of amiodarone at the rate of $28.8 \mathrm{mg}$ per hour; which resulted in the improvement of the atrial flutter. Echocardiogram done subsequently revealed severe systolic heart failure with an ejection fraction of $25 \%$. The patient lab parameters deteriorated suddenly with worsening coagulopathy and aminotransferases rising to 65 - 70 times the upper limit of normal. Highest levels of aspartate aminotransferase (AST), alanine aminotransferase (ALT) and INR were 3,000 U/L, 1,500 U/L, and 24 respectively. Trend of liver enzymes and coagulation studies are shown in Figure 1 and Figure 2. Amiodarone was discontinued on the third day. Ultrasound of the abdomen showed hepatomegaly with diffusely increased echogenicity. The patient had no urticaria, rashes nor eosinophilia. There was no significant hypotension. Retrospective review did not reveal use of alcohol or any other hepatotoxic drug besides amiodarone. Workup for viral hepatitis panel including hepatitis panel, cytomegalovirus,

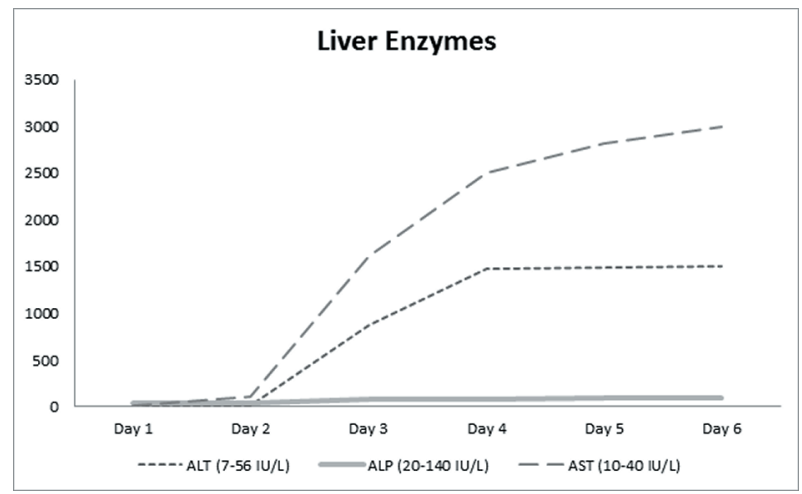

Figure 1. Trend of liver enzymes. 


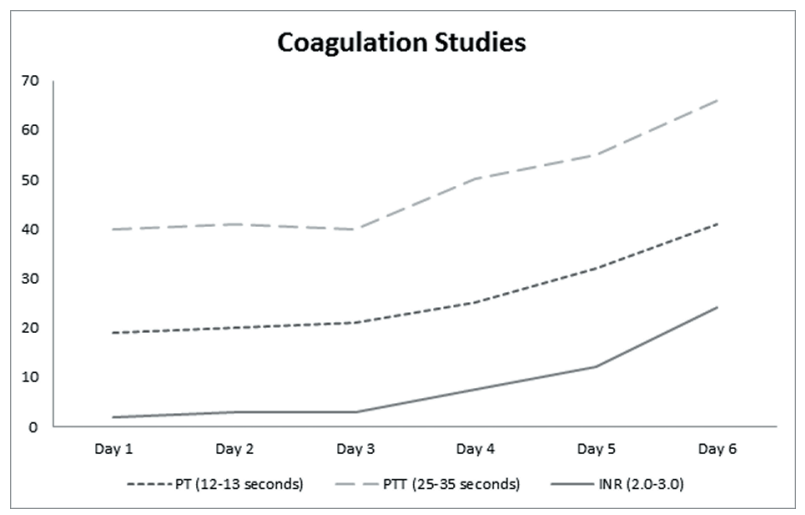

Figure 2. Trend of coagulation studies.

Epstein-Barr virus, herpes simplex virus, human immunodeficiency virus as well as blood culture and vasculitis workup were negative. His liver function continued to deteriorate and on the sixth day he developed multiple organ dysfunctions with eventual cardiac arrest and death. A diagnosis of acute fulminant liver failure secondary to amiodarone was established.

\section{Discussion}

Amiodarone is a routinely used lipophilic class III antiarrhythmic drug which is widely used in the United States in the management of ventricular and atrial arrhythmias. The drug has a half-life of up to 6 months and accumulates in tissues. It causes impairment of liver function tests with elevated transaminase levels seen in up to $15-50 \%$ of patients with chronic use. Cases of fatal acute liver failure due to amiodarone, as seen in our patient, are rarely seen [2-5].

While an exact mechanism for intravenous amiodarone induced acute liver failure has not been well defined, it is thought to be a distinct mechanism than the one causing chronic liver failure. The prevailing hypothesis of hypotensive effect is attributed to a chemical called polysorbate 80 found in intravenous preparations of amiodarone but not in oral ones [6]. Rhodes et al described a case of a patient who developed hepatic impairment with elevated transaminases within $24 \mathrm{~h}$ of starting intravenous amiodarone [7]. The liver function improved once the intravenous amiodarone was held. Interestingly, the patient was later started oral amiodarone with no signs of liver injury corroborating the hypothesis of amiodarone-induced acute liver failure secondary to a reaction caused by polysorbate 80 . A case control retrospective study by Gluck et al in 2011 including 22 cases of intravenous amiodarone induced hepatitis and 25 cases of ischemic hepatitis concluded indistinguishable features between the groups, and provide support for the hypothesis of a hypotensive effect [8].

\section{Conclusions}

The exact mechanism for intravenous amiodarone induced acute liver failure is not well defined, but the hypotensive ef- fect attributable to polysorbate 80 present in intravenous amiodarone is a compelling hypothesis. Further investigative studies are needed to elucidate the exact mechanism and identify high-risk patients. Meanwhile, it is important for clinicians to be aware of this rare but potentially fatal complication of intravenous amiodarone so that it can be withdrawn immediately at the first sign of hepatic impairment.

\section{Author Contributions}

Vijay Gayam: conception of the design, literature review, final drafting. Mazin Khalid: conception of the design, literature review, final drafting. Sumit Dahal: revision of the discussion and citation. Pavani Garlapati: obtaining data, review of the final draft. Arshpal Gill: obtaining data, review of the final draft. Alex Ragin: obtaining data, review of the final draft. Mohammad Mansour: critical review and proof reading.

\section{Conflict of Interest}

None.

\section{Informed Consent}

Informed consent was obtained from the patient's next of kin.

\section{References}

1. Amiodarone: U.S. National Library of Medicine. National Institutes of Health; Available from: https://livertox. nlm.nih.gov//Amiodarone.htm.

2. Li JG, Yang TC, Yu DM, Ren TH. Fatal acute liver failure after intravenous amiodarone administration. J Formos Med Assoc. 2015;114(3):294-296.

3. Akbal E, Batgi H, Kocak E, Canatan T, Koklu S. Lowdose amiodarone-induced fatal liver failure. Drug Chem Toxicol. 2013;36(2):261-262.

4. MacFadyen RJ, Palmer TJ, Hisamuddin K. Rapidly fatal acute amiodarone hepatitis occurring in the context of multiple organ failure. Int J Cardiol. 2003;91(2-3):245247.

5. Kalantzis N, Gabriel P, Mouzas J, Tiniakos D, Tsigas D, Tiniakos G. Acute amiodarone-induced hepatitis. Hepatogastroenterology. 1991;38(1):71-74.

6. Gough WB, Zeiler RH, Barreca P, El-Sherif N. Hypotensive action of commercial intravenous amiodarone and polysorbate 80 in dogs. J Cardiovasc Pharmacol. 1982;4(3):375-380.

7. Rhodes A, Eastwood JB, Smith SA. Early acute hepatitis with parenteral amiodarone: a toxic effect of the vehicle? Gut. 1993;34(4):565-566.

8. Gluck N, Fried M, Porat R. Acute amiodarone liver toxicity likely due to ischemic hepatitis. Isr Med Assoc J. 2011;13(12):748-752. 